\title{
Dietary Supplementation with Omega-3 Alpha- Linoleic Acid and Polyunsaturated Fatty Acids (Pufas): A New Therapeutic Protocol Additional to Scaling and Root Planing in the Treatment of Chronic Periodontitis: A 6 Months Follow Up
}

\author{
Martina Salvatorina Murgia, Simone Domenico Aspriello \\ ${ }^{1}$ Departement of Surgical Science, University of Cagliari, 09124 Cagliari, Italy
}

${ }^{2} D D S, P h D, A S D$, Dental Clinic, Pesaro, Italy

Received: February 15, 2021; Accepted: March 01, 2021; Published: April 19, 2021

“Corresponding author: Simone Domenico Aspriello, DDS, PhD, Private Dental Practice, Pesaro, Italy, E-mail: simonedomenico@yahoo.it

\begin{abstract}
Objectives: Periodontitis is a chronic inflammatory disease with a multifactorial etiology. In the literature, numerous adjuvant treatments have been introduced for the treatment of this pathology. Recently, polyunsaturated fatty acids (PUFAs) have been shown to have anti-inflammatory therapeutic properties in chronic inflammatory diseases, including periodontitis. The aim of this pilot study was to investigate the effect of 6 months dietary supplementation with Omega 3, $\alpha$-linolenic acid (ALA) and PUFAs (Freia Alfalife Farmaceutici Srl) following Scaling and Root Planning (SRP) in patients affected by chronic periodontitis.
\end{abstract}

Methods: Twenty-four participants with moderate chronic periodontitis were enrolled in our randomised, placebo-controlled trial. The control group ( $\mathrm{CG}=12$ ) was treated with SRP and given a placebo; the treatment group (TG=12) was treated with SRP and dietary supplementation of $3 \mathrm{~g}$ daily of alphalife (Freia Farmaceutici Srl). Probing depth (PD), clinical attachment level (CAL), bleeding on probing (BOP) and modified sulcus bleeding index (SBI) were recorded at baseline and after 6 months.

Results: CAL, PD, BOP and SBI were significantly improved in both groups after 6 months ( $p<0.001)$. After 6 months CAL improved significantly better in the test than in the control group $(\mathrm{p}<0.001)$, also PD in the tendency $(\mathrm{p}=0.1)$. BOP improved better in the test group after 6 months $(\mathrm{p}=$ 0.065).

Conclusion: Our results suggest PUFAs can significantly reduce periodontal parameters following SRP. Therefore, dietary supplementation may have potential benefits in the treatment of patients with chronic periodontitis.

Keywords

$\alpha$-linolenic acid (ALA);Adjuvant treatment;Chronic periodontitis;Dietary Supplements;Omega-3;Polyunsaturated fatty acids (PUFAs).

\section{Abbreviations \& Acronyms}

ALA: $\alpha$-linolenicAcid

PUFAs: Polyunsaturated Fatty Acids

SRP: Scaling and Root Planing

CG: Control Group

TG: Test Group

PD: Probing Depth

CAL: Clinical Attachment Level

BOP: Bleeding on Probing

SBI: Sulcus Bleeding Index
EPA: Eicosapentaenoic Acid

DHA: Docosahexaenoic Acid

ARA: Arachidonic Acid

\section{Introduction}

Periodontitis is a multifactorial inflammatory chronic disease that leads to the irreversible destruction of the supporting tissues (i.e., gingiva, periodontal ligament and alveolar bone) resulting in tooth loss and contribution to the development of various systemic diseases [1,2]. The Global Burden of Disease Study ranked it sixth among the most common diseases in the world, with 743 million people affected [3]. Periodontitis is the result of the unwell controlled interaction between bacterial products, proinflammatory (such us cytokines, chemokines, 


\section{Dietary Supplementation with Omega-3 Alpha-Linoleic Acid and Polyunsaturated Fatty Acids (Pufas): A New Therapeutic Protocol Additional to Scaling and Root Planing in the Treatment of Chronic Periodontitis: A 6 Months Follow Up}

Copyright:

(C) 2021 Simone Domenico A et al. arachidonic acid metabolites and proteolytic enzymes) and angiogenetic (such as VEGF, NOS) mediators and numerous cell populations [4-6]. The gold standard of periodontal therapy, both in the initial stages and during maintenance, is based on the mechanical removal of the biofilm but this is not able to lead to his absolute resolution [7-9]. Consequently, numerous alternative or supportive therapeutic options to the causal therapy of periodontitis have emerged in the literature [10-13]. The most powerful pro-resolving mediators of inflammation in chronic inflammatory diseases, including periodontitis, are resolvins, deriving from omega- 3 fatty acids, eicosapentaenoic acid (EPA; 20: 5n-3) and docosahexaenoic acid (DHA; 22: 6n-3) [14-17]. Polyunsaturated Fatty Acids (PUFAs) have more than 1 covalent double bond between carbon atoms; to this class belong omega-3, omega-6 and omega-9 $[18,19]$. Considering these potential beneficial properties, an adequate and balanced intake omega-3 alpha-linoleic acid and PUFAs is potentially beneficial for protection against chronic and metabolic diseases [19]. Due to the proven anti-inflammatory properties, some studies in the literature have experimented PUFAs dietary supplementation as an adjuvant therapy to SRP in patients with chronic periodontitis, obtaining conflicting results. Furthermore, this new approach avoids the use of non-steroidal anti-inflammatory drugs, which can be administered in a limited way and have well documented side effects [20-22]. Therefore, following the numerous works in the literature, the aim of this pilot study was to investigate and evaluate the effectiveness of a dietary supplement with $\alpha$-linolenic Omega 3 (ALA) and PUFAs (alphalifeFreiaFarmaceuticisrl, Figure 1) in addition to Scaling Root Planing (SRP) for 6 months in the treatment of chronic periodontal disease.

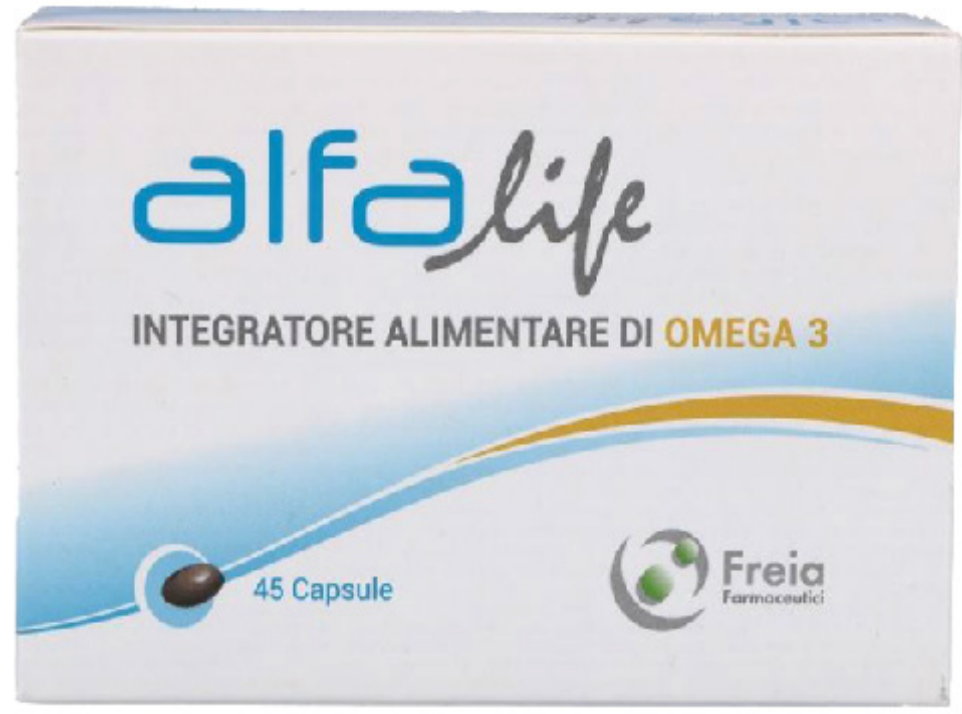

Figure 1: PUFAs dietary supplement administered in the present pilot study.

\section{Materials and Methods}

This prospective randomized blind pilot study was performed in a private clinic, Pesaro-Urbino, Marche, Italy, from February 2019 to July 2020. Thirty-six patients were assessed according to eligibility requirements (Figure 2). The inclusion criteria included:

- $\quad$ minimum number of teeth $\geq 20$;

chronic untreated periodontitis;

age between 18 to 70 years;

- $\quad$ absence of systemic pathologies that could compromise the physiological healing processes (type 2 diabetes, oncological and immunosuppressive diseases, bone turnover pathologies);

smoke;

state of pregnancy or breastfeeding; antibiotic and anti-inflammatory therapies taken within 6 months.

Patients, once the study was carefully explained, signed an informed consent form. Six patients did not meet the inclusion criteria and were excluded from the study. Finally, thirty patients were randomized into the test and control group to be received for 6 months: test group)

3g per day of alphalife (FreiaFarmaceuticisrl) $(n=15$, - $\quad 3 g$ / day of placebo $(n=15$, placebo $)$.

At the first visit, a systemic medical history was performed on all patients (with a focus on pathologies that could compromise the physiological healing processes and on any pharmacological therapies performed). A complete dental examination was then performed, with periodontal chart, radiographic examinations 


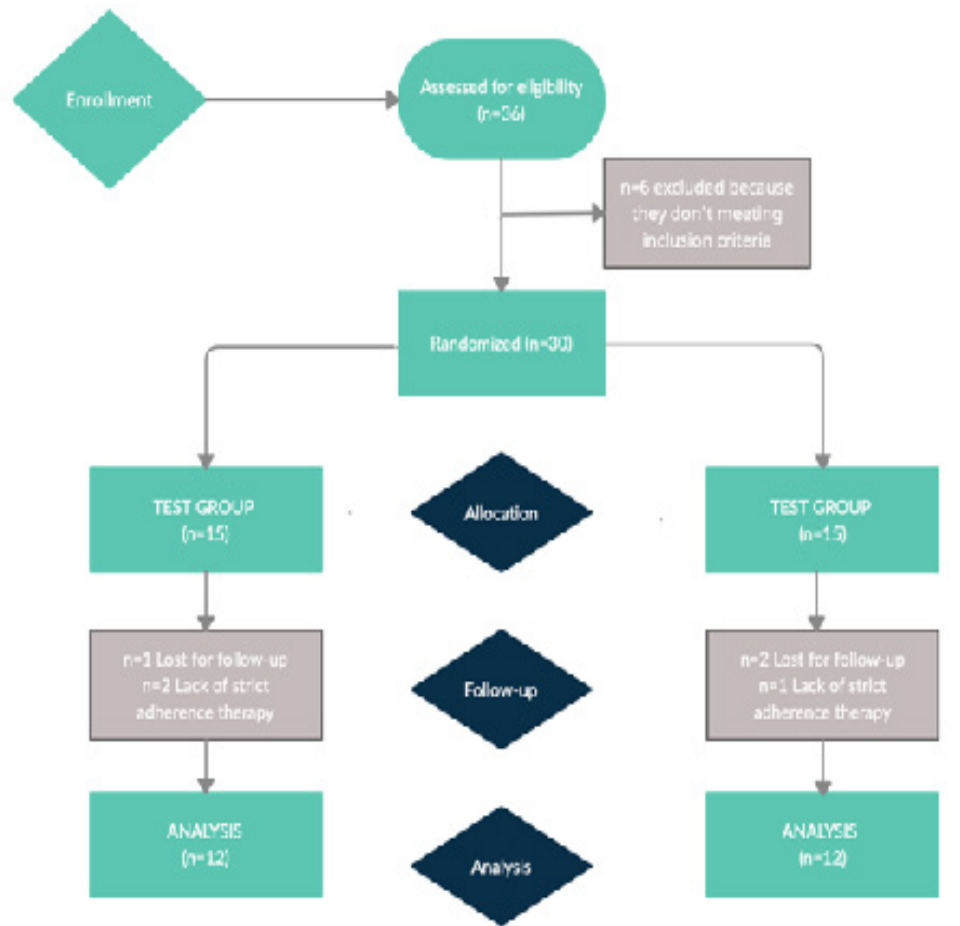

Figure 2: Flow chart of the study population.

(panoramic radiographs) and a comprehensive treatment plan. Periodontal therapy was performed on all patients and consisted of full-mouth SRP, with manual and ultrasound instruments. In addition, an important instruction in oral hygiene was performed. Clinical assessments at baseline and 6 months were recorded by the same calibrated examiner (SDA) using the same type of periodontal probe (UNC-15) and compared. Three patients in the test and three patients in the control group were lost during the follow-up or have not strictly adhered to dietary supplementation end they were excluded from data analysis. Statistical analysis was performed using SPSS software, version 22.0 (SPSS Inc., NY, U.S.A.). The null hypothesis is that there were no statistically significant differences between the two groups for parameters PD, AL, BOP and SBI at the start of the study.

Table 1: Demographics of subject population and baseline periodontal parameters.

\begin{tabular}{|c|c|c|c|}
\hline & Test & Control & $\mathrm{p}$ Value \\
\hline Age & $50.4 \pm 5.9$ & $50.8 \pm 6.7$ & \\
\hline Men & 7 & 6 & \\
\hline Women & 5 & 6 & \\
\hline PD & $3.2 \pm 0.4$ & $3.2 \pm 0.5$ & 0.967 \\
\hline CAL & $3.4 \pm 0.3$ & $3.4 \pm 0.4$ & 0.948 \\
\hline BOP (\%) & $16.6 \pm 4$ & $16.7 \pm 5.6$ & 0.726 \\
\hline SBI (\%) & $18.6 \pm 6.4$ & $18.2 \pm 5.5$ & 0.870 \\
\hline
\end{tabular}

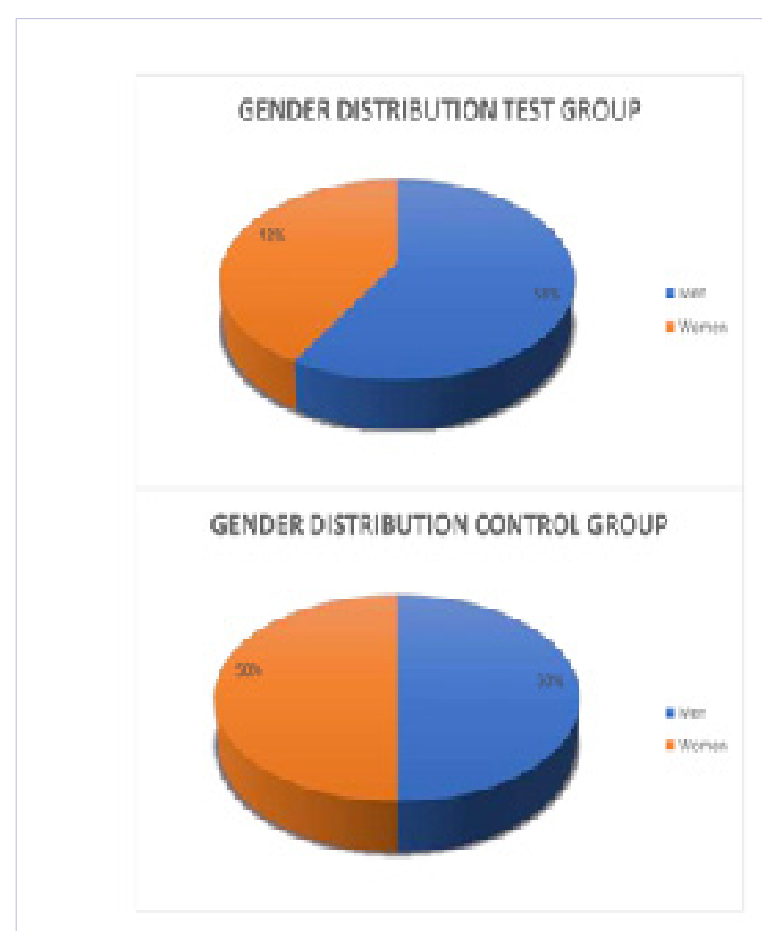

Figure 2: Gender distribution of the test and control group. 


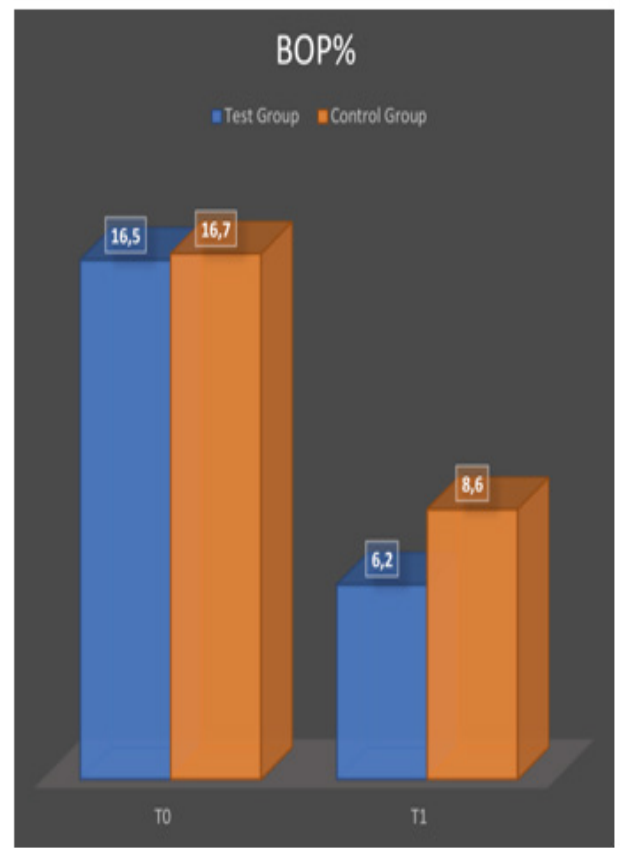

Figure 4: The categorical distribution of the BoP $\%$ for each group at $\mathrm{T} 0$ and $\mathrm{T} 1$.

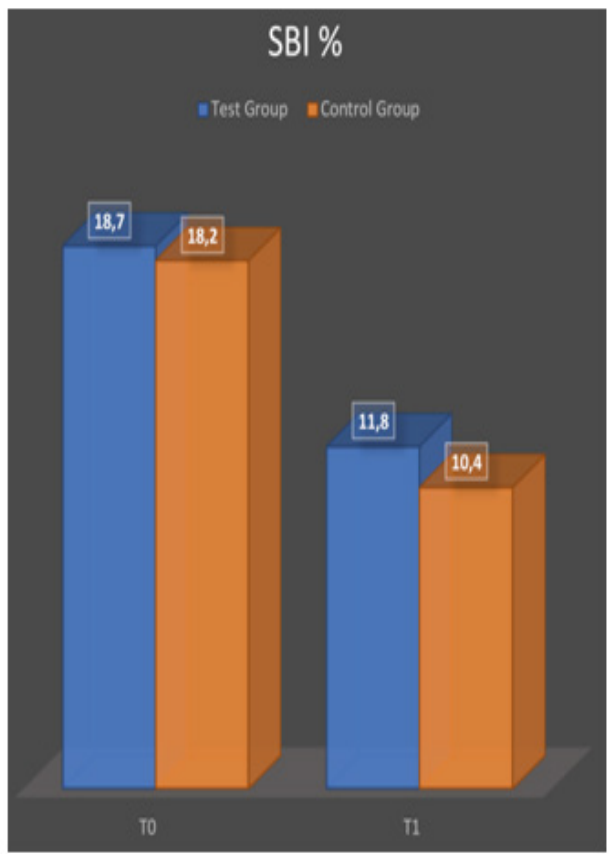

Figure 5: The categorical distribution of the SBI \% for each group at T0 and T1.

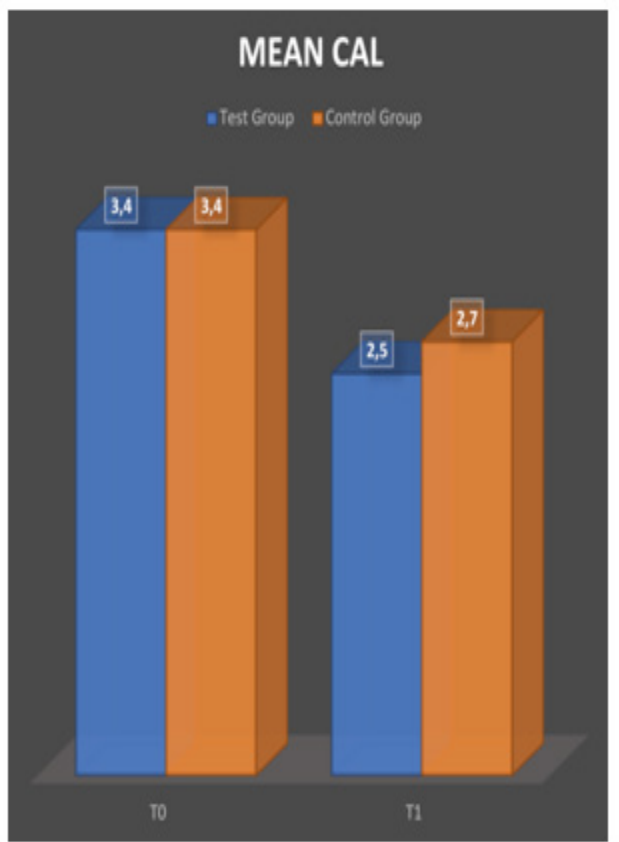

Figure 6: The categorical distribution of the mean CAL for each group at $\mathrm{T} 0$ and $\mathrm{T} 1$.

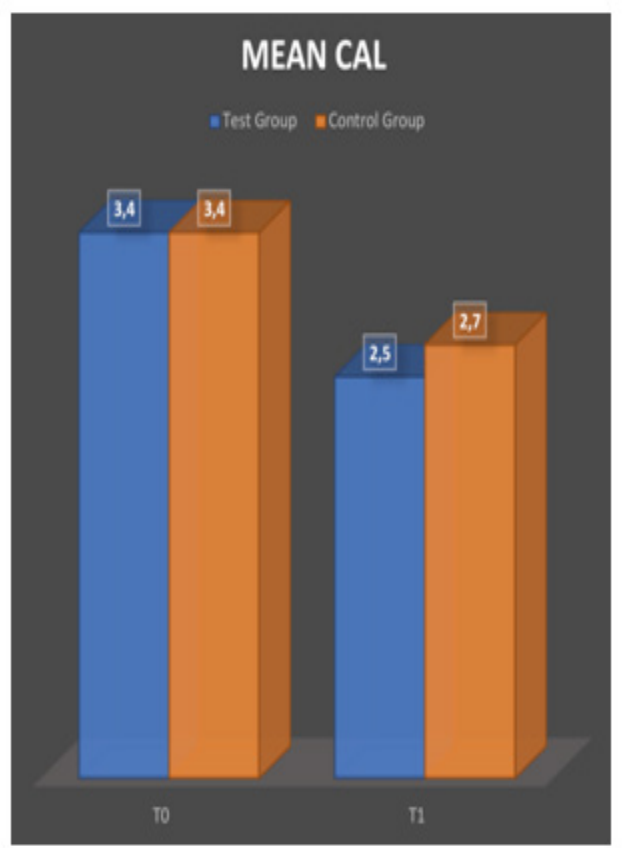

Figure 7: The categorical distribution of the mean PD for each group at $\mathrm{T} 0$ and $\mathrm{T} 1$.

Table 2:Full-mouth values for periodontal measurements at baseline (T0), and after treatment (T1).

\begin{tabular}{|c|c|c|c|}
\hline BoP (\%) & Baseline (T0) & 6 Months (T1) & Differences (T0-T1) \\
\hline Test & $16.5 \pm 4.2$ & $6.2 \pm 3.1$ & $10.3 \pm 3.2$ \\
\hline Control & $16.7 \pm 5.4$ & $8.6 \pm 3.6$ & $8.1 \pm 4.1$ \\
\hline
\end{tabular}

Citation: Martina Salvatorina Murgia, Simone Domenico Aspriello. (2021) Dietary Supplementation with Omega-3 Alpha-Linoleic Acid and Polyunsaturated Fatty Acids (Pufas): A New Therapeutic Protocol Additional to Scaling and Root Planing in the Treatment of 
Dietary Supplementation with Omega-3 Alpha-Linoleic Acid and Polyunsaturated Fatty Acids (Pufas): A New Therapeutic Protocol Additional to Scaling and Root Planing in the Treatment of Chronic Periodontitis: A 6 Months Follow Up
Copyright:

(c) 2021 Simone Domenico A et al.

\begin{tabular}{|c|c|c|c|}
\hline SBI (\%) & \multicolumn{3}{|l|}{} \\
\hline Test & $18.7 \pm 6.4$ & $11.8 \pm 4.3$ & $6.8 \pm 5.1$ \\
\hline Control & $18.2 \pm 5.5$ & $10.4 \pm 4.2$ & $7.7 \pm 5.4$ \\
\hline Mean PD (mm) & $2.1 \pm 0.2$ & $2.2 \pm 0.3$ \\
\hline Test & $3.2 \pm 0.4$ & $2.3 \pm 0.4$ & $2.3 \pm 0.4$ \\
\hline Control & $3.2 \pm 0.5$ & $2.5 \pm 0.3$ & $2.6 \pm 0.1$ \\
\hline Mean CAL (mm) & $3.4 \pm 0.3$ & $2.7 \pm 0.3$ & $2.8 \pm 0.3$ \\
\hline Test & $3.4 \pm 0.4$ & & \\
\hline Control & & & \\
\hline
\end{tabular}

\section{Discussion}

Omega- 6 and omega-3 cannot be synthesized by humans or other higher animals [19]. In the body, arachidonic acid (ARA, n 6), EPA and DHA, derive from these fatty acids, play a significant role in the homeostasis regulation $[6,19]$. Numerous studies have found that Omega-3s of marine origin have antiarrhythmic [23], anti-inflammatory [24, 25], antithrombotic, antihypertensive, anti-atherosclerotic properties [26-29], they also promote endothelial function and can reduce blood triglyceride levels [30]. Furthermore, numerous studies show that omega-3 contributes to the development and integrity of neurons in the brain, thanks to the protective antioxidant effect on cell membranes and to the potential neurochemical mechanisms directly related to Alzheimer's disease and dementia [31-33]. This randomized controlled interventional study, masked as an examiner, conducted for duration of 6 months showed a significant improvement in PD, BOP and CAL in both groups compared to baseline measurements. These periodontal parameters are strictly dependent on tissue inflammation; therefore, their improvement indicates that there has been a resolution of the periodontal inflammation. There was a statistically significant difference when the two groups were compared. This is in agreement with the study by Sharkawyet al. [34], which however, in addition to the administration of the PUFAs, added a daily administration of $81 \mathrm{mg}$ of aspirin. In a recent randomized controlled trial similar to the present work, Kujur et al. showed a significant reduction in PD and CAL at 1 and 3 months, respectively, in the test group (SRP and PUFAs $500 \mathrm{mg}$ ) compared to the control one (only SRP), demonstrating the beneficial effects of dietary PUFAs supplementation [35]. These results are in agreement with those obtained by Deore et al. in which 60 patients were divided into two groups: the test one underwent SRP and daily administration of PUFAs while the control one only SRP [36]. Another recent randomized clinical trial obtained a statistically significant reduction in BOP and an improvement in CAL at three months in the test group $(\mathrm{n}=16)$ treated with SRP and daily PUFA supplementation compared to the control one $(n=14$, treated with only SRP [37]. On the other hand, in the study by Keskineret al., very similar to ours, thirty healthy subjects suffering from chronic periodontitis were divided into two groups $(\mathrm{n}=15)$. The test group was treated with SRP and daily low-dose PUFAs and the control group with SRP and placebo. The results showed that both groups had significant changes in clinical parameters from baseline but without significant differences between the groups. This would suggestthat the systemic benefits of dietary omega-3 PUFAs may not be translated into periodontal health [38]. Martinez et al. also evaluated daily dietary supplementation based on omega-3, EPA and DHA for 4 months in the test group affected by chronic periodontitis in addition to SRP, compared to the control group treated with only SRP and placebo [39]. Also in this study, periodontal examination included PD, CAL, BOP, and plaque index. The results showed that dietary supplementation with omega-3 had no effect on the clinical outcome of the treatment, since periodontal parameters were not significantly different between the test and placebo groups at baseline. Generally, we can affirm that most of the studies examined have shown a significant improvement in periodontal indices following dietary supplementation based on PUFAs [34-36,40]. Differences in sample size, PUFAs dose and treatment duration (Table 3 ) could justify the incomplete homogeneity of the results examined. Another important fact that is not expressed in the studies cited is the overall diet of the patients enrolled in the study, which, in the opinion of the Authors, plays a decisive role in the clinical outcomes and in the patient's systemic health. One aspect to note is that low-dose aspirin was not administered in this study, as it may have anti-inflammatory action and may act as a confounding factor. The strength of the study also lies in the fact that all intraoral examinations and periodontal treatment were performed by the same calibrated examiner. SRP for all patients was performed in the same setting with the same ultrasound and manual devices. Patient compliance was checked at all downsizing and maintenance recall visits; in addition, oral hygiene instructions were strengthened at all visits. Therefore, we can propose that supplementation with PUFAs may constitute a valid support therapy in the treatment of periodontal disease.

\section{Conclusion}

Food supplementation with PUFAs in addition to SRP can reduce gingival inflammation by modulating the inflammatory cytokine cascade. Therefore, this can be used as an adjuvant treatment for chronic periodontitis. After 6 months of intervention, patients who received PUFAs supplements compared with placebo had significantly better periodontal parameters following SRP.Finally, 
these results suggest that omega- 3 could be a valid support in the treatment of periodontal disease. Further multicenter and controlled clinical trials would be needed to verify whether a dietary supplement based on PUFAs and omega-3s may be able to play a preventive role in the pathogenesis of periodontitis (in terms of delayed onset, reduction in the severity of pathological outcomes).

\section{References}

1. Papapanou PN, Sanz M, Buduneli N, Dietrich T, Feres M, Fine DH et al. Periodontitis: Consensus report of workgroup 2 of the 2017 World Workshop on the Classification of Periodontal and Peri-Implant Diseases and Conditions. J Periodontol. 2018;89 Suppl 1:S173-S182.

2. Aspriello SD, Zizzi A, Tirabassi G, Buldreghini E, Biscotti T, Faloia E et al. Diabetes mellitus-associated periodontitis: differences between type 1 and type 2 diabetes mellitus. J Periodontal Res. 2011;46(2):164-169.

3. Tonetti MS, Jepsen S, Jin L, Otomo-Corgel J. Impact of the global burden of periodontal diseases on health, nutrition and wellbeing of mankind: A call for global action. J Clin Periodontol. 2017;44(5):456-462.

4. Hajishengallis G, Chavakis T, Lambris JD. Current understanding of periodontal disease pathogenesis and targets for host-modulation therapy. Periodontol 2000. 2020;84(1):14-34.

5. Aspriello SD, Zizzi A, Lucarini G, Rubini C, Faloia E, Boscaro M, Tirabassi G, Piemontese M. Vascular endothelial growth factor and microvessel density in periodontitis patients with and without diabetes. J Periodontol. 2009;80(11):1783-9.

6. Van Dyke TE, Sima C. Understanding resolution of inflammation in periodontal diseases: Is chronic inflammatory periodontitis a failure to resolve? Periodontol. 2020;82(1):205-213.

7. Lucarini G, Zizzi A, Aspriello SD, Ferrante L, Tosco E, Lo Muzio, L et al. Involvement of vascular endothelial growth factor, CD44 and CD133 in periodontal disease and diabetes: an immunohistochemical study. J Clin Periodontol. 2009;36(1):3-10.

8. Bastendorf KD, Strafela-Bastendorf N, Lussi A. Mechanical Removal of the Biofilm: Is the Curette Still the Gold Standard? Monogr Oral Sci. 2021;29:105-118.

9. Dental Scaling and Root Planing for Periodontal Health: A Review of the Clinical Effectiveness, Cost-effectiveness, and Guidelines. Ottawa (ON): Canadian Agency for Drugs and Technologies in Health. 2016.

10. Biloklytska G, Braun I. Combined Usage of Diode Laser "Granum" and "Emdogain" (Straumann) During Surgical Phase in Treatment of Generalized Periodontitis. J Dent Oral Disord Ther; 2014;2(3):1-7.

11. Kothiwale S, Kella M, Hombal L, Rathore A. Evaluation of sodium bicarbonate as an adjunct to non surgical periodontal therapy and its effect on oxidative stress: A clinico-biochemical study. J Dent Oral Disord Ther. 2014;2(2):5.

12. Mykytenko AO, Yankovskiy DS, Dyment GS, Beregova TV, Neporada KS. "Simbiter Omega" Multiprobiotic: A New Aid in Treatment of Generalized Chronic Periodontitis. J Dent Oral Disord Ther. 2015;3(1):1-6.

13. Farjana HN, Anand, Chandrasekaran SC. "Resolvins: A novel theraputic approach in treating periodontal disease. Indian J Multidiscip Dent. 2015;5:10-4

14. Innes JK, Calder PC. Omega-6 fatty acids and inflammation.
Prostaglandins Leukot Essent Fatty Acids. 2018;132:41-48.

15. Van Dyke TE. Pro-resolving mediators in the regulation of periodontal disease. Mol Aspects Med. 2017;58:21-36.

16. Chiang N, Serhan CN. Specialized pro-resolving mediator network: an update on production and actions. Essays Biochem. 2020;64(3):443462.

17. Vardar-Sengul S, Buduneli E, Turkoglu O, Buduneli N, Atilla G, et al. The effects of selective COX-2 inhibitor/celecoxib and omega-3 fatty acid on matrix metalloproteinases, TIMP-1, and laminin-5gamma2-chain immunolocalization in experimental periodontitis. J Periodontol. 2008;79(10):1934-41.

18. Román GC, Jackson RE, Gadhia R, Román AN, Reis J. Mediterranean diet: The role of long-chain $\omega-3$ fatty acids in fish; polyphenols in fruits, vegetables, cereals, coffee, tea, cacao and wine; probiotics and vitamins in prevention of stroke, age-related cognitive decline, and Alzheimer disease. Rev Neurol (Paris). 2019;175(10):724-741.

19. Saini RK, Keum YS. Omega-3 and omega- 6 polyunsaturated fatty acids: Dietary sources, metabolism, and significance - A review. Life Sci. 2018 ;203:255-267.

20. Reddy MS, Geurs NC, Gunsolley JC. Periodontal host modulation with antiproteinase, anti-inflammatory, and bone-sparing agents. A systematic review. Ann Periodontol. 2003;8(1):12-37.

21. Salvi GE, Lang NP. Host response modulation in the management of periodontal diseases. J Clin Periodontol. 2005;32 Suppl 6:108-29.

22. Joseph A, Nalini HE, Kumar PA, Devi R. Pharmacotherapy for host modulation in periodontal disease: A review. J Indian Acad Dent Spec Res. 2015;2:35-39.

23. Tribulova N, Bacova B, Benova T, Knezl V, Barancik M, Slezak J. Omega-3 Index and Anti-Arrhythmic Potential of Omega-3 PUFAs. Nutrients. 2017;9(11):1191.

24. Wall R, Ross RP, Fitzgerald GF, Stanton C. Fatty acids from fish: the antiinflammatory potential of long-chain omega-3 fatty acids. Nutr Rev. 2010;68(5):280-289.

25. Calder PC. Omega-3 fatty acids and inflammatory processes: from molecules to man. Biochem Soc Trans. 2017 ;45(5):1105-1115.

26. Serhan CN, Chiang N, Van Dyke TE. Resolving inflammation: dual antiinflammatory and pro-resolution lipid mediators. Nat Rev Immunol. 2008;8(5):349-61.

27. Ajith TA, Jayakumar TG. Omega-3 fatty acids in coronary heart disease: Recent updates and future perspectives. Clin Exp Pharmacol Physiol. 2019;46(1):11-18.

28. Endo J, Arita M. Cardioprotective mechanism of omega-3 polyunsaturated fatty acids. J Cardiol. 2016;67(1):22-7.

29. Ferguson B, Bokka NR, Maddipati KR, Ayilavarapu S, Weltman R, Zhu L, et al. Distinct Profiles of Specialized Pro-resolving Lipid Mediators and Corresponding Receptor Gene Expression in Periodontal Inflammation. Front Immunol. 2020;11:1307.

30. Colussi G, Catena C, Novello M, Bertin N, Sechi LA. Impact of omega-3 polyunsaturated fatty acids on vascular function and blood pressure: Relevance for cardiovascular outcomes. Nutr Metab Cardiovasc Dis. 2017;27(3):191-200.

31. Burckhardt M, Herke M, Wustmann T, Watzke S, Langer G, Fink A. Omega-3 fatty acids for the treatment of dementia. Cochrane Database Syst Rev. 2016;4(4):CD009002 
32. Song C, Shieh $\mathrm{CH}$, Wu YS, Kalueff A, Gaikwad S, Su KP. The role of omega-3 polyunsaturated fatty acids eicosapentaenoic and docosahexaenoic acids in the treatment of major depression and Alzheimer's disease: Acting separately or synergistically? Prog Lipid Res. 2016;62:41-54.

33. Ajith TA. A Recent Update on the Effects of Omega-3 Fatty Acids in Alzheimer's Disease. Curr Clin Pharmacol. 2018;13(4):252-260.

34. Sharkawy H, Aboelsaad N, Eliwa M, Darweesh M, Alshahat M, Kantarci A, et al. Adjunctive treatment of chronic periodontitis with daily dietary supplementation with omega-3 Fatty acids and low-dose aspirin. J Periodontol. 2010;81(11):1635-1643.

35. Kujur SK, Goswami V, Nikunj AM, Singh G, Bandhe S, Ghritlahre H. Efficacy of omega 3 fatty acid as an adjunct in the management of chronic periodontitis: A randomized controlled trial. Indian J Dent Res. 2020;31(2):229-235.

36. Deore GD, Gurav AN, Patil R, Shete AR, Naiktari RS, Inamdar SP. Omega 3 fatty acids as a host modulator in chronic periodontitis patients: a randomised, double-blind, palcebo-controlled, clinical trial. J Periodontal Implant Sci. 2014;44(1):25-32.
37. Stańdo M, Piatek P, Namiecinska M, Lewkowicz P, Lewkowicz N. Omega-3 Polyunsaturated Fatty Acids EPA and DHA as an Adjunct to Non-Surgical Treatment of Periodontitis: A Randomized Clinical Trial. Nutrients. 2020;12(9):2614.

38. Keskiner I,Saygun I, BalV,Serdar M, Kantarci A. Dietary supplementation with low-dose omega-3 fatty acids reduces salivary tumor necrosis factor- $\alpha$ levels in patients with chronic periodontitis: a randomized controlled clinical study. J Periodontal Res. 2017;52(4):695-703.

39. Martinez GL, Koury JC, Brito F, Fischer RG, Gustafsson A, Figueredo $\mathrm{CM}$. The impact of non-surgical periodontal treatment on serum levels of long chain-polyunsaturated fatty acids: a pilot randomized clinical trial. J Periodontal Res. 2014;49(2):268-74.

40. Aspriello SD, Murgia MS, Casu C, Coiana C, Fais S, Orrù G et al. Effect of 3 months dietary supplementation with omega- $3 \alpha$-linolenic acid (ala) and polyunsaturated fatty acids (PUFAS) (Alphalife Freia Framaceutici Srl) following scaling and root planing: a pilot study. Journal of Osseointegration. 2020;12:613-614. 